\title{
La nueva normalidad en el Camino de Santiago: reflexiones para el futuro
}

\section{Lucrezia Lopez* / Rubén Camilo Lois González}

Universidade de Santiago de Compostela, Departamento de Xeografía, Praza da Universidade 1, 15703 Santiago de Compostela, España

Recibido: 8 de marzo de 2021 / Aceptado: 14 de junio de 2021

\begin{abstract}
Resumen
La COVID-19 ha alterado las relaciones globales y los equilibrios territoriales, y no menos afectada ha resultado la movilidad, cuyas dinámicas han sido reconfiguradas. El turismo ha sido el sector económico más castigado por la pandemia. La presente propuesta pone el foco de atención en una realidad turística de envergadura internacional, tal y como es el Camino de Santiago, basado en desplazamiento y movilidad. El objetivo es contribuir al debate actual acerca de los impactos de la COVID-19 en el Camino de Santiago y avanzar reflexiones para su futura recuperación. Por ello, se adopta una metodología cualitativa interpretativa que pone en relación estudios anteriores y herramientas de planificación y gestión turística. Los resultados indican un comportamiento diferente a corto, medio y largo plazo, y se considera que el Camino volverá a ser el benchmark de los itinerarios de peregrinación.
\end{abstract}

\section{Palabras clave}

Camino de Santiago; COVID-19; nueva normalidad.

\section{The new normal on the Way of Saint James: Reflections for the future}

\begin{abstract}
COVID-19 altered global relations and territorial balances, and mobility was no less affected, as its dynamics were reconfigured. Tourism was the economic sector hardest hit by the pandemic. This proposal focuses on a tourist reality of international impotance, such as the Way of Saint James, based on travel and mobility. The aim is to contribute to the current debate on the impacts of COVID-19 on the Way of Saint James and to advance reflections for its future recovery. For this reason, a qualitative interpretive methodology is adopted that links previous studies and tourist planning and management tools. The results indicate a different behaviour in the short, medium and long term, and it is considered that the Way will once again become the benchmark for pilgrimage itineraries.
\end{abstract}

Keywords

Way of Saint James; COVID-19; new normality.

JEL Codes: 018, R11, Z12, Z3.

* L. Lopez: lucrezia.lopez@usc.es (autora para correspondencia), R. C. Lois González: rubencamilo.lois@usc.es 


\section{Introducción}

La pandemia causada por la COVID-19 ha alterado las relaciones globales y los equilibrios territoriales, y no menos afectada ha resultado la movilidad. No hemos vivido solo una limitación a la movilidad internacional, sino que hemos conocido una inesperada limitación a la movilidad a escala local, que ha reducido o ha anulado nuestros desplazamientos diarios. La salida del confinamiento de la primera ola, a finales de junio de 2020, significó una lenta y desigual recuperación de la "libertad de movimiento" y, con ella, un pautado ingreso en la, así llamada, "nueva normalidad", una expresión que se ha difundido en los últimos meses para indicar el conjunto de medidas y hábitos que deberíamos aprender para convivir con la pandemia. Estamos viendo como "la nueva normalidad" no supone la salida definitiva de la pandemia, cuya duración sigue siendo incierta (a pesar de las vacunas y de la inmunidad de rebaño), sino que alude a la convivencia con ella para poder desempeñar las actividades diarias, resaltando así las capacidades de adaptación propias de toda comunidad.

Como consecuencia de este hecho histórico, se ha reconfigurado el escenario de la movilidad de negocio, reforzando modalidades de trabajo no presencial y el e-commerce. El deseo de recuperar el tiempo perdido animó los primeros desplazamientos turísticos durante el verano de 2020 (primer verano de la pandemia). El turismo ha sido el sector económico más castigado por la pandemia, independientemente del segmento, precisamente por basarse en el movimiento de personas (Febrero Paños y Bermejo Patón, 2021). Se ha demostrado la fragilidad de los territorios dependientes sobre todo de la actividad turística; asimismo, una de las primeras enseñanzas de esta crisis sanitaria, y consecuente caída turística, fue confirmar que la economía de un territorio debe contar con sectores diversificados (The World Tourism Organization [UNWTO], 2020). De hecho, el cierre causado por la explosión de la pandemia ha desencadenado un balance de las prácticas turísticas, en modo espe-cial de las de masa, que anteponían un uso intensivo del espacio turístico a su valor cualitativo y experiencial. En tal sentido, según Thomas Friedman (2020), la pandemia ha marcado un hito histórico, que permite hablar del mundo A. C. (antes del coronavirus) y del mundo D. C. (después del coronavirus).

A partir de estas premisas, y con una mirada más atenta al sector turístico, esta propuesta pone el foco de atención en una realidad turística de envergadura internacional: el Camino de Santiago. Si hasta hace poco más de un año la preocupación era el overtourism y sus impactos en la experiencia del peregrinación, hoy debemos de partir de la actual crisis turística mundial para analizar los impactos de la COVID-19 en el camino de peregrinación (Lopez y Lois González, 2020). El Camino de Santiago es un "mundo", en cuanto que se caracteriza por su propia humanidad (es decir, por los diferentes colectivos que lo viven). Debido a esto, requiere una atención especial, y por tanto para su reapertura se han diseñado una serie de medidas que podrían tener impactos en sus dinámicas, ritos y prácticas. El 2021 es una cita importante, y esperada por muchos, debido a que, después de once años, el 2021 es Año Santo Compostelano (o Año Jacobeo, ya que el 25 de julio, día del Apóstol, cae en domingo). Al asumir que este Año Santo 2021 no cumplirá con las expectativas, de forma excepcional se ha declarado Año Santo también el 2022, durante el cual se espera poder recuperar los beneficios que ya se dan por perdidos en este 2021.

Asimismo, esta propuesta quiere contribuir al debate acerca de los impactos de la COVID-19 en el Camino de Santiago a través de una perspectiva histórica que analiza pasado, presente y futuro del itinerario jacobeo. Desde el punto de vista metodológico, adoptamos un enfoque cualitativo-interpretativo, presentamos una revisión de estudios anteriores sobre el Camino, así como los instrumentos de planificación diseñados antes y durante la explosión de la pandemia. Siguiendo una línea del tiempo, introducimos la caracterización del Camino antes de la pandemia, resaltando su dimensión experiencial y vivencial. Continuamos presentando la nueva normalidad a lo largo del Camino, valorando las posibles repercusiones de la limitación a la movilidad internacional. Como resultado, avanzamos algunas reflexiones sobre el futuro del Camino en la nueva normalidad, teniendo en cuenta los efectos de las limitaciones de movilidad y las limitaciones sanitarias prescritas para evitar el contagio (edad, grupos de riesgos, etcétera). 


\section{Materiales y métodos}

Según J. Creswell (2014), para el diseño de una investigación es necesario establecer un marco general que aborde las diferentes facetas del estudio, desde la evaluación de las ideas generales, pasando por los datos para, luego, llegar al análisis y a la evaluación. Entre el variado mosaico de perspectivas de investigación, cada una con sus presupuestos teórico-conceptuales y metodológicos (Patton, 2002), hemos optado por una metodología cualitativa interpretativa, a través de la que se intenta dar sentido a fenómenos referidos a un caso de estudio (Creswell, 1998; Denzin y Lincoln, 2018; Vasilachis, 2006). Igualmente, la estructura de la investigación pretende responder a los tres elementos estructurales indicados por J. Mason (1996): 1) definir la esencia de la investigación; 2) definir los datos; y 3) indicar métodos de análisis y explicación.

En primer lugar, hemos revisado y hemos analizado estudios anteriores relativos al Camino de Santiago antes de la pandemia a fin de presentar un estado del arte y con ello entender el alcance de las peregrinaciones jacobeas antes de la pandemia. A través de esta revisión, hemos puesto de manifiesto los factores de éxito de un camino de peregrinación que supera fronteras intercontinentales. La revisión bibliográfica introduce también un aspecto cuantitativo, en concreto hace referencia a la explotación da la serie estadística publicada por la Catedral de Santiago de Compostela (que registra el número de peregrinos que recogen la Compostela ${ }^{1}$ en la Oficina de Recepción de Peregrinos de la Diócesis de Santiago). Los informes publicados por la Catedral de Santiago en su página web presentan diferentes tipos de información. En este ejercicio interpretativo no menos relevantes han sido las fuentes referidas a las herramientas de planificación y gestión del turismo, en general, y del Camino, en lo específico. A través de estas, destacamos el papel vertebrador que desempeña el Camino para la política turística gallega, y como consecuencia la atención recibida ya en las primeras semanas de la explosión de la crisis. Esta lectura transversal introduce a corto y a medio plazo nuevos patrones de comportamiento, y avanza nuevos escenarios motivacionales que sumarán complejidad al mundo del Camino.

\section{El Camino de Santiago antes de la pandemia de la COVID-19}

La peregrinación es un ámbito interdisciplinar y un proceso histórico y geográfico cuyo desarrollo está también relacionado con factores económicos, políticos, antropológicos y, como hemos tenido ocasión de descubrir recientemente, con cuestiones sanitarias que afectan a la movilidad internacional (Mróz, 2021). De hecho, los estudios de peregrinación están estrechamente relacionados con cuestiones de movilidad, siendo la peregrinación un desplazamiento hacia y a los sitios (Coleman y Eade, 2004). Se trata de una movilidad compleja y fructuosa, resultante de la interacción de varios fenómenos que generan relaciones espaciales y culturales a diferentes escalas y, por esta razón, es preciso analizar cuestiones asociadas a distancia y comportamiento (Cohen, 1992; Collins-Kreiner, 2010; Digance, 2003; Eade y Sallnow, 1991; Stoddard y Morinis, 1997). Las peregrinaciones son también consideradas las primeras formas de desplazamientos turísticos (Apollo, Wengel, Schänzel y Musa, 2020; Collins-Kreiner, 2010, 2015; Timothy y Olsen, 2006). Este carácter móvil explica la caída de los movimientos de peregrinación hacia las principales metas católicas durante y después de la primera ola de la pandemia (Mróz, 2021).

Una de las peregrinaciones de más éxito de los últimos años es el Camino de Santiago, primer Itinerario Cultural Europeo y una de las rutas de peregrinación medieval más importantes del mundo. La expresión "camino" se utiliza para referirse a una red de itinerarios que numerosos peregrinos recorren para llegar a la ciudad de Santiago de Compostela, meta final. El centro histórico urbano de Santiago y algunos de los Caminos han sido nombrados Patrimonio de la Humanidad por la UNESCO. Primero, en 1993 el Camino Francés fue declarado Patrimonio Mundial de la Humanidad; luego en 2015 los Caminos del Norte recibieron el mismo galardón internacional. Desde el año 1993, el Camino ha sido fuertemente impulsado por el Gobierno regional, por lo que en los últimos años ha habido un crecimiento continuo en el número de peregrinos que llegan a Santiago, pasando de 99.436 en 1993 a 347.578 en 2019 (Oficina del Peregrino). Hasta la interrupción de la serie histórica causada por la CO-

${ }^{1}$ El certificado otorgado por la Iglesia al caminante que, según la tradición, ha recorrido andando más de cien kilómetros en la ruta hacia Santiago y doscientos en bicicleta o a caballo. 
VID-19, los datos publicados por la catedral de Santiago referidos a los últimos diez años muestran el aumento de peregrinos que recogen su Compostela.

Con esta tendencia evidentemente positiva, y su relevancia en la planificación turística gallega, el Plan director e estratéxico do Camiño de Santiago en Galicia 2015-2021 (Xunta de Galicia, 2015) fija unas directrices a favor de la conservación y de la protección del Camino (con un presupuesto total de $56.119 .805 €$ y un presupuesto estimado por peregrino de 70,66 €). Esta herramienta de gestión servía sobre todo en vista del Xacobeo 21, por el cual la estimación total era de 464.000 peregrinos, con un gasto medio diario de $44 € /$ día y $264 €$ /semana (Xunta de Galicia, 2015). Estas previsiones ya positivas fueron mejoradas en el Plan estratéxico do Xacobeo (Xunta de Galicia, 2019), según el cual los peregrinos previstos iban a subir a 502.606. Todas esas expectativas motivaron la puesta en marcha de proyectos colaterales, animando a muchos empresarios del Camino a renovar sus negocios (Lopez y Lois González, 2020).

Según consta en el cuestionario previo a la entrega de la Compostela, los peregrinos pueden elegir entre ocho opciones de rutas (Camino Francés, Camino Portugués, Camino Inglés, Vía de la Plata, Camino del Norte, Camino Primitivo y Camino Portugués de la Costa). Hasta el comienzo de la pandemia, el Camino Francés era la ruta más transitada, y por ello empezaban a manifestarse problemas relativos a su capacidad de carga y a la saturación de los albergues públicos (Fraternidad Internacional del Camino de Santiago, 2014; Martín-Duque, 2017; Porcal Gonzalo, Díez Angulo e Íñiguez de Heredia, 2012). Siempre hasta el comienzo de la pandemia, la segunda ruta más recorrida era una de las tres variantes del Camino Portugués, es decir, el Camino Portugués de la Costa, opción introducida por la Catedral en 2016. En cuatro años, el número de peregrinos a lo largo de esta variante superó otras rutas como el Camino del Norte, el Camino Primitivo y el Camino Inglés. Podríamos, así, asumir que una consecuencia de la masificación de la ruta principal es la progresiva diversificación de los itinerarios. El mismo Plan director e estratéxico do Camiño de Santiago en Galicia 2015-2021 (Xunta de Galicia, 2015) contempla como línea estratégica la potenciación de todas las rutas de peregrinación a Santiago de Compostela mediante la dinamización de los diferentes Caminos y la difusión de los valores de cada uno de ellos, fomentando así la singularidad de aquellas rutas poco exploradas (Martínez Roget, Castro Domínguez y Fraiz Brea, 2018).

El éxito del Camino, reflejado en cifras positivas hasta el año 2019, puede tener su clave de interpretación en su carácter inclusivo, resumido en: 1) mercados internacionales; 2) confesiones y religiones; 3) movilidad; 4) motivaciones; 5) caminonización ${ }^{2}$ de las industrias creativas. Esos aspectos están relacionados entre ellos y se retroalimentan. Factores diferentes han contribuido a la internacionalización del Camino, como confirma la diversificación de la nacionalidad de los mercados emisores (Lois González y Santos, 2015) (Figura 1). En los últimos diez años, se aprecia un crecimiento en el mercado estadounidense, cuyo número de peregrinos supera las cifras de los países vecinos, es decir, Francia y Portugal (Martínez Roget et al., 2018). También ha aumentado el número de peregrinos procedentes de Brasil, mientras que en el mercado asiático Corea casi ha duplicado su presencia en cuatro años, pasando de 4.535 peregrinos en 2016 a 8.224 en 2019 (Tabla 1).

Esta visibilidad internacional ha transformado el Camino en una ruta multiconfesional y multirreligiosa a lo largo de la cual peregrinos y turistas de distintas confesiones comparten un único espacio (Lois González, 2013; Santos y Lois González, 2011). El mismo Plan estratéxico do Xacobeo 2021 (Xunta de Galicia, 2019) reconoce la multiculturalidad del Camino como valor estratégico y punto de fuerza a partir del cual reforzar la imagen exterior de la ruta jacobea. A lo largo de esta, los no creyentes pueden disfrutar estando cerca de la naturaleza; de hecho, uno de los atributos más valorados del Camino es el paisaje (Xunta de Galicia, 2019), gracias al cual se propicia también la paz espiritual (Farias et al., 2018; Moscarelli, Lopez y Lois González 2020).

Teniendo en cuenta la complejidad del Camino, adoptamos un doble enfoque: movilidad física y movilidad "metafísica" (o introspectiva). En lo que se refiere a la movilidad física, esta se caracteriza por ritmos lentos y finalizados a lo largo del tiempo (Lois González y Lopez, 2012). Para ello, los peregrinos disponen de distintas modalidades de desplazamiento (a pie, a caballo o en bicicleta). La movi-

\footnotetext{
2 Esta expresión recuerda el término caminonization, a través del cual el antropólogo Peter Margry (2015) hace referencia a la internacionalización de modelos y valores asociados al Camino de Santiago. Si bien el concepto definido por el antropólogo indica, en primera instancia, las implicaciones territoriales y sociales del Camino, esta expresión también se emplea para indicar el "efecto Camino" en distintas esferas de la vida cultural.
} 
lidad física lenta es, a la vez, consecuencia y causa de la movilidad interior e introspectiva. Es consecuencia, en la medida en que la movilidad introspectiva alimenta el deseo de ponerse en camino; es causa, en cuanto refuerza una dimensión espiritual no solo para los caminantes espirituales sino también para los que peregrinan por otras motivaciones, y que de forma inesperada descubren un viaje más profundo (Chemin, 2011; Doi, 2011).

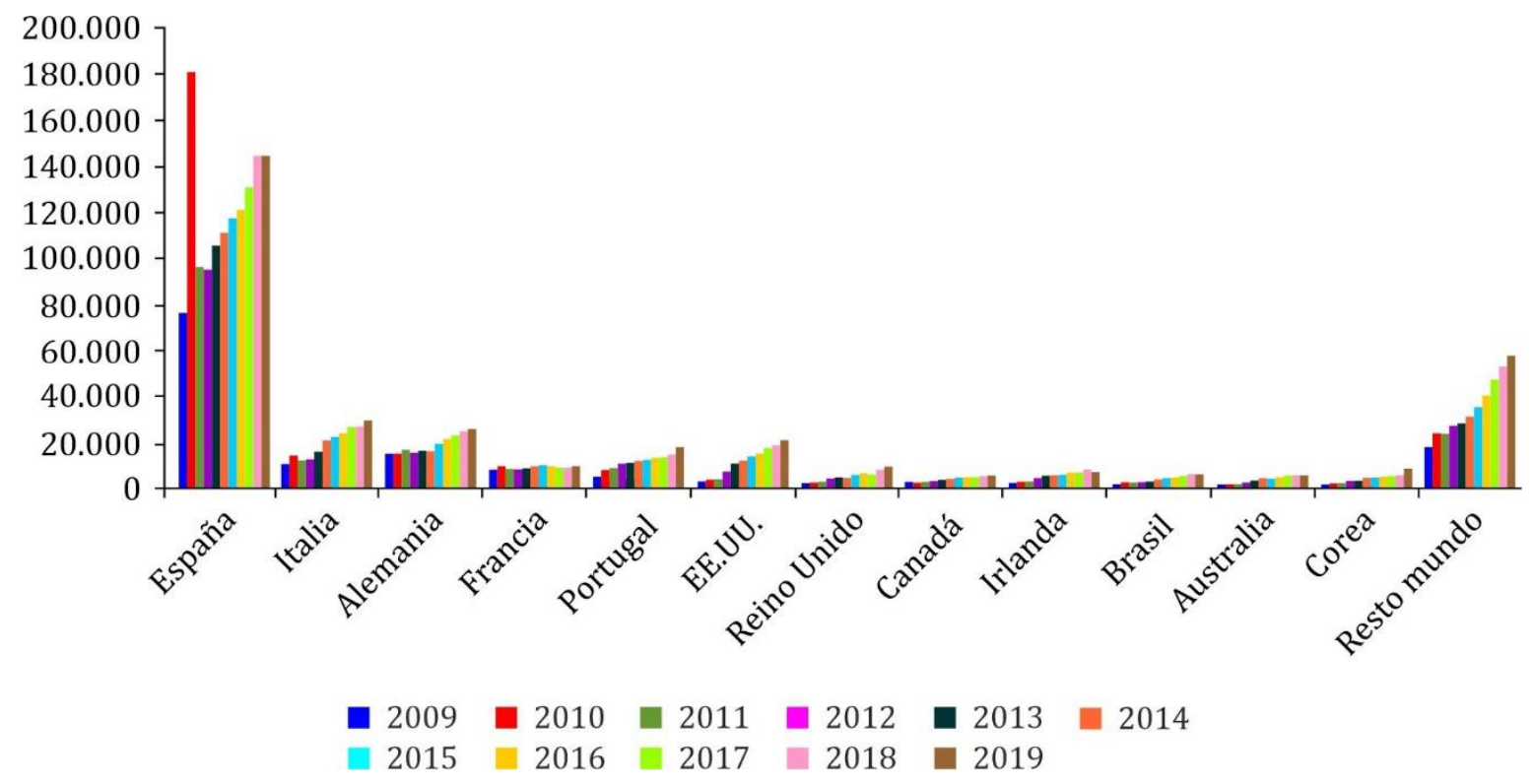

Figura 1. Evolución del número de peregrinos según las principales nacionalidades. Fuente: elaboración propia a partir de los datos de la Oficina del Peregrino.

Tabla 1. Datos estadísticos relativos a la procedencia de los peregrinos según las principales nacionalidades

\begin{tabular}{cccccccc}
\hline & España & Italia & Alemania & Francia & Portugal & EE.UU. & $\begin{array}{c}\text { Reino } \\
\text { Unido }\end{array}$ \\
\hline 2009 & 79.007 & 10.341 & 14.789 & 7.459 & 4.854 & 2.540 & 1.700 \\
2010 & 188.089 & 14.222 & 14.503 & 9.140 & 7.786 & 3.334 & 2.031 \\
2011 & 97.822 & 12.183 & 16.596 & 8.166 & 8.649 & 3.726 & 2.389 \\
2012 & 95.275 & 12.404 & 15.620 & 8.121 & 10.329 & 7.071 & 3.758 \\
2013 & 105.891 & 15.621 & 16.203 & 8.305 & 10.698 & 10.125 & 4.207 \\
2014 & 113.655 & 20.254 & 16.348 & 9.348 & 11.578 & 11.578 & 4.396 \\
2015 & 122.420 & 22.151 & 18.873 & 9.916 & 12.481 & 13.670 & 5.417 \\
2016 & 124.230 & 23.944 & 21.220 & 8.868 & 13.245 & 15.236 & 6.050 \\
2017 & 132.479 & 27.073 & 23.227 & 8.835 & 12.940 & 17.522 & 5.768 \\
2018 & 144.141 & 27.009 & 25.296 & 8.775 & 14.413 & 18.582 & 7.624 \\
2019 & 146.350 & 28.749 & 26.167 & 9.248 & 17.450 & 20.652 & 9.248 \\
\hline & Canadá & Irlanda & Brasil & Australia & Corea & Resto del mundo & Total \\
\hline 2009 & 2.194 & 1.722 & 1.248 & 1.015 & 1.079 & 17.929 & 145.877 \\
2010 & 1.877 & 2.296 & 2.121 & 1.191 & 1.455 & 24.090 & 272.135 \\
2011 & 2.362 & 2.677 & 1.983 & 1.352 & 1.740 & 23.721 & 183.366 \\
2012 & 2.904 & 3.844 & 2.229 & 1.885 & 2.493 & 26.555 & 192.488 \\
2013 & 3.373 & 5.012 & 2.431 & 3.098 & 2.774 & 28.142 & 215.880 \\
2014 & 3.576 & 5.022 & 3.272 & 3.792 & 3.842 & 31.322 & 237.983 \\
2015 & 4.201 & 5.367 & 3.938 & 3.856 & 4.073 & 36.153 & 262.516 \\
2016 & 4.354 & 6.537 & 4.365 & 4.441 & 4.534 & 40.830 & 277.854 \\
2017 & 4.612 & 6.643 & 5.113 & 4.890 & 5.106 & 46.828 & 301.036 \\
2018 & 5.027 & 7.548 & 5.601 & 5.220 & 5.665 & 52.477 & 327.378 \\
2019 & 5.279 & 6.826 & 6.025 & 5.301 & 8.224 & 58.059 & 347.578 \\
\hline
\end{tabular}

Fuente: elaboración propia a partir de los datos de la Oficina del Peregrino. 
Esa movilidad introspectiva está relacionada con otro aspecto que ha contribuido a la transformación poscontemporánea del Camino: la variedad de motivaciones (Nilsson y Tesfahuney, 2019). N. L. Frey (1998) ya había afirmado que, debido a los diferentes perfiles de peregrinos, era difícil generalizar sus motivaciones. En esta misma línea, K. Doi (2011) reconoció la dificultad en caracterizar los perfiles de peregrinos debido a la variedad de procedencia y motivaciones. Desde luego, la motivación es relevante para los estudios turísticos en cuanto que explica comportamientos y expectativas (Losada Sánchez, Alén González y Domínguez Vila, 2014). En el caso del Camino de Santiago, estamos antes una situación compleja. Por un lado, varios estudios sociológicos y antropológicos adoptan sus criterios metodológicos para investigar los perfiles motivacionales de los peregrinos a lo largo del Camino (Chemin, 2011; Kurrant, 2019). Entre ellos, C. Kurrant (2019) adopta una metodología cualitativa social para analizar los perfiles a través de las biografías de los peregrinos alemanes, y señala motivaciones vinculadas a una dimensión personal (balance de vida, crisis, transición y nuevo comienzo).

Por otro lado, existe la serie histórica producida por la Catedral, cuyas estadísticas siguen siendo referencia a la hora de aproximarse al número y al perfil de peregrinos (aunque con unas limitaciones). Los primeros datos recogidos se remontan al año 1989, cuando el 83,5\% de los peregrinos que llegó a Santiago declaró haber realizado el viaje por motivos religiosos, el $12,6 \%$ por motivos religioso-culturales y solo el 1,5\% por motivos culturales. Veinte años después (en 2009), las motivaciones religiosas son indicadas por el $42,6 \%$ de los peregrinos, el $48,2 \%$ declara razones mixtas relacionadas con motivaciones religiosas, y el 9,2\% afirma haber hecho el Camino sin ninguna motivación religiosa. En $2019^{3}$, no se registran cambios significativos, ya que un 48,7\% indica haber hecho el Camino por motivos religiosos y otros, un 40,3\% exclusivamente religiosos, y un 10,98\% no religiosos (Figura 2).

1989

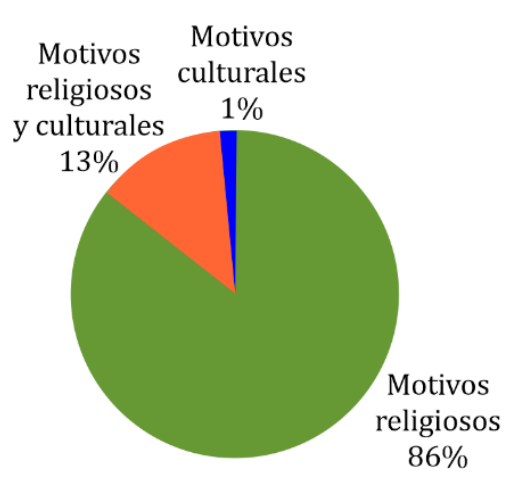

2009

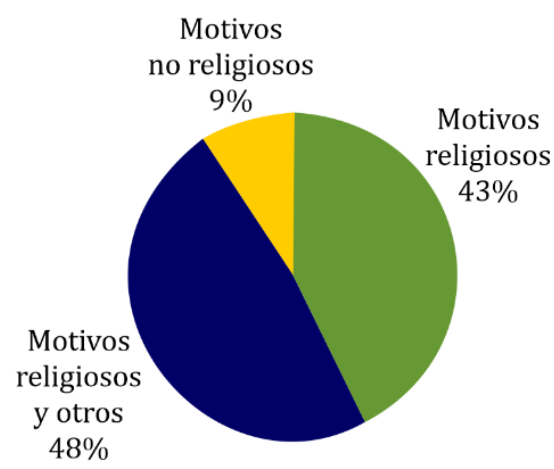

2019

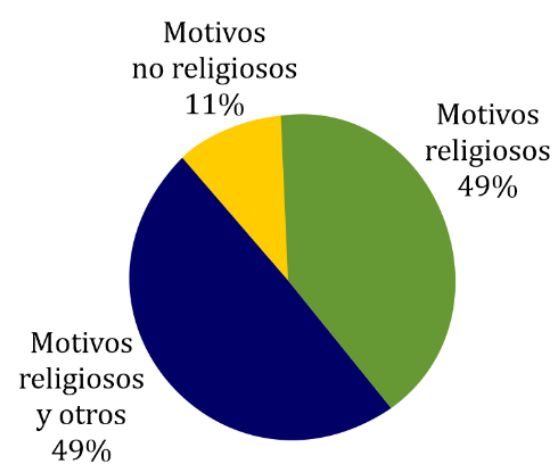

Figura 2. Evolución de la motivación de los peregrinos. Fuente: elaboración propia a partir de los datos de la Oficina del Peregrino.

Si bien estas estadísticas son de interés general, hace una década el Observatorio del Camino de Santiago se encargó de definir con precisión el perfil de los peregrinos y planificar, gestionar y mejorar la oferta y la experiencia de los caminantes. Las motivaciones mostradas por el Observatorio eran más variadas en comparación con las propuestas por el Arzobispado, y los datos revelaban que la mayoría de los peregrinos se desplazaba hacia Compostela por una motivación espiritual, pero no religiosa (Centro de Estudios Turísticos [CETUR] y Xacobeo S.A., 2007-2010). Solo en los Años Santos las razones religiosas se aproximaban a ese valor, pero destacaban muchas otras razones para justificar el inicio del viaje, como la riqueza de la naturaleza, el paisaje, la historia, el arte o, simplemente, los deportes o la diversión (Tabla 2).

En su estudio, F. Cazaux (2011) afirma que los peregrinos definen su viaje como espiritual porque de este modo establecen una distancia con la Iglesia (cuya historia y autoridad a menudo se cuestionan), pero al mismo tiempo afirman "creer en algo". De hecho, explica que si, por un lado, la religión alude a un conjunto de reglas y a la sumisión entendida como corolario de la religión, la definición de espiritual alude a un peregrino poscontemporáneo capaz de poner en cuestión el mundo que le rodea.

${ }^{3}$ Se toma como último año el 2019 a raíz de la pandemia del 2020 y sus consecuencias en el número de peregrinos. 
El significado de fe en el Camino se ha diversificado en épocas recientes; si bien la espiritualidad sigue siendo una de las principales motivaciones para recorrer el Camino y visitar Santiago, la peregrinación jacobea es hoy una ocasión de redescubrimiento de uno mismo, una experiencia que permite vivir una relación equilibrada con la naturaleza, una forma diferente de vivir la vida social, el tiempo y el entorno paisajístico (CETUR y Xacobeo S.A., 2007-2010; Lois González, Castro Fernández y Lopez, 2016; Moscarelli et al., 2020). El paso de la religiosidad a la espiritualidad y la importancia cada vez mayor de la dimensión espiritual refuerzan el carácter inclusivo del Camino y, desde el punto de vista económico, permiten abrir nuevos horizontes de mercado.

Tabla 2. Principales motivos para hacer el Camino

\begin{tabular}{lcccc}
\hline & $2007(\%)$ & $2008(\%)$ & $2009(\%)$ & $2010(\%)$ \\
\hline Patrimonio histórico-artístico & 25,8 & 25,0 & 23,9 & 21,2 \\
Patrimonio natural & 39,3 & 33,7 & 36,0 & 31,3 \\
Motivación espiritual & 50,3 & 53,1 & 49,4 & 48,5 \\
Motivación religiosa & 38,1 & 38,3 & 39,5 & 47,6 \\
Cultura popular & 16,2 & 15,7 & 14,6 & 14,7 \\
Deporte & 22,8 & 19,8 & 20,8 & 19,7 \\
Diversión & 17,1 & 14,8 & 19,2 & 17,1 \\
Otro & 7,2 & 8,0 & 9,0 & 8,8 \\
\hline
\end{tabular}

Fuente: elaboración propia a partir de los datos de CETUR y Xacobeo S.A. (2007-2010).

Recientemente, se han publicado los resultados del proyecto El impacto del Covid-19 en el perfil del peregrino, coordinado por D. Riveiro García (2021). Entre los distintos cambios en el perfil del peregrino que los investigadores han destacado, señalamos aquí el aspecto motivacional, pues parece confirmarse la tendencia a la baja de la motivación religiosa, junto a una reducción del carácter espiritual y experiencial del Camino. Los peregrinos entrevistados responden más a un perfil lúdico, haciendo del Camino una alternativa vacacional con amplia oferta de recursos y al aire libre. De hecho, la principal motivación para hacer el Camino son el arte y la cultura, seguidos por vacaciones y turismo.

Efectivamente, a lo largo del Camino se ofrece una lectura renovada de las antiguas obligaciones de visitar iglesias y monasterios, que se han convertido en recursos para el turismo cultural y patrimonial. La oferta del Camino es variada, sobre todo desde que, en los últimos años, se le reconocen valores asociados al goce de paisajes y de elementos medioambientales que ayudan a la salud física y espiritual (Lois González y Santos, 2015; Milani, 2001; Sobrino y López, 2006; Santos y Lois González, 2011). A tal propósito, los paisajes del Camino están asumiendo el carácter de paisajes terapéuticos, en los cuales ambiente físico y construido, condiciones sociales y percepciones humanas producen una atmósfera propicia para la curación (Gesler, 1996). Esta reinterpretación puede ayudar a comprender la práctica de continuar hasta Fisterra como expresión de un "pluralismo espiritual", ya que estos caminantes refuerzan el carácter individual de la espiritualidad poscontemporánea, desvinculada del poder de la Iglesia Católica (la ruta no está reconocida oficialmente por la Iglesia) (Margry, 2015).

No menos importante es el hecho de que el Camino se haya convertido en un tema creciente en las industrias creativas, incluida la literatura, el cine y las artes, en respuesta a la tendencia a la especialización en las artes y en las humanidades (Lopez y Lois González, 2020). Encuentros, emociones, miedos y sucesos inspiran al peregrino, sea escritor, artista o dibujante, que aprovecha esos tipos de espacios para compartir su geografía personal con su público (Lopez, 2020). Motivaciones y actitudes poscontemporáneas están contribuyendo a hacer del Camino un Leitmotiv de las industrias creativas y, al mismo tiempo, producen nuevas formas de explorar y acercarse a la dimensión subjetiva de los peregrinos.

Las resultantes combinaciones de estos cinco factores responsables de la inclusividad del Camino crean un espacio internacional y multiconfesional, en el cual peregrinos y turistas interactúan para co-crear una identidad y una personalidad de la ruta y reforzar la hipermovilidad que la pandemia global de la COVID-19 detuvo abruptamente. 


\section{La nueva normalidad del Camino de Santiago}

La crisis sanitaria y económica ha generado un frenesí en la planificación turística, pues ha sido necesario actualizar medidas y estrategias de gestión y dar respuestas a la urgencia de planificar el futuro de la economía del turismo, lejos de prácticas y realidades pasadas que causaron overtourism, gentrificación y, evidentemente, la dependencia del turismo (UNWTO, 2020). Por ello, se ha confiado mucho en este momento de cambio para mirar hacia un posible decrecimiento turístico (Fletcher, Murray, Blázquez-Salom y Blanco Romero, 2020; Harvey, 2020).

Después de más de un año desde la explosión de esta crisis, convivimos con miedos e incertidumbres, intentando hacer un balance del pasado para sacar alguna enseñanza útil que nos permita hacer previsiones a corto, medio y largo plazo (Febrero Paños y Bermejo Patón, 2021). En pleno Año Xacobeo 2021-2022, considerado como oportunidad para la dinamización económica gallega (sobre todo para el rural), por el cual se preveía un crecimiento del Producto Interior Bruto (PIB) gallego de un 0,9\% y la creación de 11.500 empleos a tiempo completo (Xunta de Galicia, 2019), hay que convivir con una realidad económica complicada e incierta.

El informe Previsións de impacto do COVID-19 no sector turístico galego (Clúster Turismo de Galicia, 2020), publicado en abril de 2020, analiza una primera caracterización de la demanda turística: limitaciones legales a la libre circulación, dependencia a corto plazo del turismo de proximidad, crisis de confianza y miedo a viajar, con la consecuente reducción de conexiones internacionales, anunciando así nuevos hábitos de consumo. En el caso del Camino, el primero y el principal problema ha sido la progresiva desaparición de peregrinos, ya que, como consecuencia del cierre durante los meses de abril y mayo de 2020, no se ha registrado ningún peregrino recogiendo su Compostela y se ha interrumpido la serie histórica de la Oficina del Peregrino. Según el ya mencionado informe (Clúster Turismo de Galicia, 2020), en un escenario post-COVID 2020, es decir, con el levantamiento del confinamiento desde finales de abril o comienzos de mayo, y con la recuperación del mercado doméstico, las llegadas alcanzarían alrededor de 131.062 peregrinos, pero la realidad ha sido más dura y los peregrinos que, finalmente, han recogido su Compostela en el año 2020 han sido 53.905 (Oficina del Peregrino).

El cierre del Camino y la progresiva desaparición del peregrino han tenido sus impactos en la "microeconomía del Camino", es decir, en aquel conjunto de actividades económicas que florecieron gracias a la ruta jacobea, aprovechando un comportamiento de consumo basado en bienes y servicios locales y generando procesos virtuosos para la economía local (Martínez Roget et al., 2018); una microeconomía caminera que redistribuye riqueza a lo largo de la ruta y que contribuye a crear puestos de trabajo en actividades tradicionales, como alojamiento y manutención, junto a otras actividades emergentes más bien vinculadas a la logística y al transporte. De hecho, la creación de estos empleos estaba generando impactos socioeconómicos positivos. En su estudio, M. Fernández Fernández, D. Fernández Méndez y D. Riveiro García (2021) analizan el efecto multiplicador del Camino en la creación de puestos de trabajos directos e indirectos.

Entre las actividades símbolos del Camino están los albergues (públicos y privados), lugares de encuentro y de condivisión. El Plan director e estratéxico do Camiño de Santiago en Galicia 2015-2021 (Xunta de Galicia, 2015) defiende el impulso a la creación de nuevos albergues en zonas donde la oferta privada es escasa o nula. Los albergues han visto peligrar su supervivencia a raíz de la COVID-19, sobre todo en los casos de albergues rehabilitados y reformados bajo la expectativa del escenario positivo del Xacobeo 2021-2022. Para aquellos que han decidido reabrir, sea a finales de junio de 2020 (para los pocos peregrinos que quedaron "atrapados en el Camino") o en los meses sucesivos, ha sido necesario adaptarse a las indicaciones sanitarias, siendo la primera de ellas el respeto del distanciamiento social y, en segundo lugar, la obligación de reducir la capacidad de alojamiento, haciendo más difícil su supervivencia por los costes de adaptación (Figura 3). A esas medidas, hay que sumar otro concepto en los presupuestos de gestión: los costes derivados de las medidas de limpieza e higiene. A tal fin, la Federación Española de Amigos del Camino (FEAACS, 2020) informó de que el sector de los albergues requería la colaboración de instituciones públicas y un paquete de medidas específicas para mantener su viabilidad.

Precisamente para hacer frente a la crisis del sector, en mayo de 2020 la Consellería de Cultura e Turismo de la Xunta de Galicia hace público un Plan de reactivación dos sectores cultural e turís- 
tico fronte aos efectos derivados da COVID-19 (Xunta de Galicia, 2020), dotado de una inversión de 26,8 millones de euros, repartidos como sigue: 10,2 millones para el sector cultural, 11 millones para el sector turístico, reforzados con 5,6 millones de euros a través de medidas transversales enmarcadas en el Xacobeo 2021-2022. También pone en marcha el programa Camino Seguro, con el objetivo de promover una peregrinación adaptada a la situación sanitaria a través de un protocolo integral de actuación en los itinerarios jacobeos en Galicia. Como indica en su página web (https://www.caminodesantiago.gal/es/caminoseguro), el programa incluye medidas como las siguientes: limitaciones de aforo de entre $50 \%$ y $75 \%$, elaboración de guías y manuales de apoyo, formación para más de 300 albergueros, ayudas para la adaptación de los establecimientos a las medidas higiénico-sanitarias, servicio de asesoramiento para los negocios y un nuevo sistema online de reservas de albergues. El desarrollo de nuevas plataformas tecnológicas es importante para facilitar las reservas en los albergues, informando en todo momento a los peregrinos de las plazas disponibles. De cara el futuro, esta práctica podría llevar a fomentar dinámicas de colaboración a diferentes escalas para detectar posibles necesidades en el trazado. En definitiva, el éxito de este programa depende de la capacidad de establecer la máxima coordinación entre los distintos organismos gestores interesados en la reactivación de la peregrinación jacobea.

\section{MEDIDAS DE AUTOPROTECCIÓN DE PEREGRINOS}

Mantener las medidas de distanciamiento social tanto en el Camino como al atravesar núcleos poblados y en los albergues.

Extremar la higiene personal, con uso frecuente de jabón de manos y solución hidroalcohólica después de tocar superficies susceptibles de transmitir el virus.

PREVENCIÓN EN LOS ALBERGUES

Equipamientos, exterior, acceso, registro, dormitorio, zonas comunes, etc.

MEDIDAS DE PROTECCIÓN PARA EL PERSONAL Y LOS HOSPITALEROS

Evitar el saludo con contacto físico, desinfectar. No compartir equipos de trabajo, usar EPI, etc.

Figura 3. Medidas de prevención presentadas por los municipios del Camino. Fuente: Protocolo de actuación para los albergues de peregrinos ante la COVID-19 y elaboración propia.

\section{El futuro pospandemia del Camino (reflexiones y perspectivas)}

Quizás hayamos aprendido que no existen fórmulas mágicas o algoritmos que puedan generar gráficos de la evolución de los flujos de peregrinos, y, por tanto, las previsiones estadísticas parecen poco acertadas. Además, también hemos aprendido que existen diferentes escenarios (a corto, medio y largo plazo) que dibujan una realidad diferente.

\subsection{La hipermovilidad y la humanidad del Camino, ¿volverán a ser las mismas?}

La movilidad es consecuencia de la distancia que, en el caso de la peregrinación, se distingue en psicológica o absoluta. En el año 1970, el geógrafo Waldo Tobler afirmó que: "todas las cosas están relacionadas entre sí, pero las cosas más próximas en el espacio tienen una relación mayor que las distantes" (Tobler, 1970, p. 236). A partir de esta ley, la interacción entre los lugares debería perder intensidad al aumentar de la distancia (distance decay). En el caso de las relaciones espaciales de las peregrinaciones, la distance decay pierde su valor (Stoddard y Morinis, 1997) y se trata la distancia también desde un punto de vista relativo y psicológico, que puede incluso llegar a reforzar el deseo de peregri- 
nar. De hecho, la distancia de un lugar sagrado determina el comportamiento del fiel y las relaciones territoriales. Por ejemplo, en el imaginario cristiano las peregrinaciones que comportan un desplazamiento más largo son también las peregrinaciones que más "recompensan"; esta imagen positiva de la distancia se funda en las dificultades y amenazas que se le suele atribuir.

Los datos referidos a la procedencia de los peregrinos revelan una contracción de los mercados intercontinentales. Por ejemplo, el total de peregrinos estadounidenses llegados durante el 2020 fue de 438, y 343 en el caso de Brasil. Las restricciones a la movilidad dibujan una realidad formada por peregrinos españoles (36.965), seguidos por peregrinos portugueses (2.949), y el mercado italiano mantiene el tercer lugar (2.694), como pasaba antes de la pandemia (Oficina del Peregrino, 2021) (Tabla 3). A escala europea, se redujo el número de peregrinos a lo largo de los itinerios transfronterizos (Camino de Santiago, Via Francígena, etcétera), puesto que los peregrinos optaron por itinerarios más bien locales y nacionales (Mróz, 2021). Quizás, también a medio plazo habrá más peregrinos europeos que asiáticos o americanos, pero eso no significa que el Camino no vuelva a ser el paradigma de la movilidad, física y metafísica. Como en el pasado, el peregrino podrá volver a ser el auténtico protagonista de la recuperación del Camino.

Tabla 3. Datos estadísticos relativos a la procedencia de los peregrinos según las principales nacionalidades en 2020

\begin{tabular}{|c|c|c|c|c|c|c|c|}
\hline & España & Italia & Alemania & Francia & Portugal & Estados Unidos & Reino Unido \\
\hline Enero & 658 & 173 & 98 & 40 & 79 & 72 & 28 \\
\hline Febrero & 1.142 & 164 & 183 & 37 & 466 & 70 & 38 \\
\hline Marzo & 801 & 52 & 201 & 30 & 94 & 76 & 31 \\
\hline Abril & - & - & - & - & - & - & - \\
\hline Mayo & - & - & - & - & - & - & - \\
\hline Junio & 11 & & & & & & \\
\hline Julio & 7.859 & 321 & 336 & 161 & 303 & 43 & 63 \\
\hline Agosto & 15.168 & 1.203 & 642 & 428 & 732 & 84 & 134 \\
\hline Septiembre & 7.275 & 411 & 479 & 336 & 662 & 41 & 136 \\
\hline Octubre & 3.825 & 311 & 330 & 386 & 590 & 51 & 116 \\
\hline Noviembre & 181 & 58 & 50 & 95 & 18 & & 25 \\
\hline Diciembre & 45 & 1 & 6 & 10 & 5 & 1 & 2 \\
\hline \multirow[t]{2}{*}{ Total } & 36.965 & 2.694 & 2.325 & 1.523 & 2.949 & 438 & 573 \\
\hline & Canadá & Irlanda & Brasil & Australia & Corea & Resto del mundo & Total \\
\hline Enero & 15 & 14 & 60 & 34 & 364 & 364 & 1.999 \\
\hline Febrero & 36 & 68 & 49 & 16 & 394 & 413 & 3.076 \\
\hline Marzo & - & 46 & 26 & - & 69 & 284 & 1.710 \\
\hline Abril & - & - & - & - & - & - & - \\
\hline Mayo & - & - & - & - & - & - & - \\
\hline Junio & & & & - & & 1 & 12 \\
\hline Julio & & & 24 & - & - & 642 & 9.752 \\
\hline Agosto & & 44 & 65 & - & - & 1.312 & 19.812 \\
\hline Septiembre & & 29 & 69 & - & - & 1.003 & 10.441 \\
\hline Octubre & 37 & 26 & 39 & - & - & 707 & 6.418 \\
\hline Noviembre & 6 & 8 & 10 & 5 & 5 & 125 & 586 \\
\hline Diciembre & & & 1 & 1 & 8 & 19 & 99 \\
\hline Total & 94 & 235 & 343 & 56 & 840 & 4.870 & 53.905 \\
\hline
\end{tabular}

Fuente: elaboración propia a partir de los datos de la Oficina del Peregrino.

Además de los peregrinos, otro colectivo que es parte de la humanidad del Camino está representado por los hospitaleros, cuyos perfiles también están en riesgo, primero si se tiene en cuenta su carácter internacional, y en segundo lugar poque entre ellos también hay personas mayores, a las cuales se les desaconseja ponerse en camino por razones de salud. Así que a corto y a medio plazo su perfil podría verse afectado y sufrir algunas modificaciones. Desde luego, la cuestión de la edad concierne a todos los peregrinos, ya que en los meses de verano de 2020 el porcentaje de caminantes con más de 60 años es inferior con respecto a los años anteriores (Oficina del Peregrino, 2020). Esta varia- 
ción se debe a una mayor sensibilidad y a un aumento de riesgos y complicaciones en el caso de contraer la infección por COVID-19.

\section{2. ¿Qué impactos podrá tener el distanciamiento social en las relaciones sociales a lo largo del Camino?}

Teniendo en cuenta las medidas de prevención al contagio impuestas por las instituciones (reflejadas en normativas y protocolos de actuación) y los rasgos del Camino, en la Figura 4 avanzamos unos posibles efectos del distanciamiento social en dos dimensiones del Camino: las relaciones sociales, y la reflexión e introspección. El distanciamiento afecta a las relaciones sociales en la medida en que se impusieron limitaciones a los espacios comunes en los albergues y se redujeron los contactos sociales a lo largo del Camino. El resultado de estas restricciones es "menos communitas", es decir, menos cohesión social. El concepto de communitas fue elaborado por V. Turner y E. Turner (1978) para referirse al sentido de cohesión de una sociedad religiosa, también garantizada por la legitimización de las instituciones y por los productos culturales. A lo largo de estos años, este concepto sufrió una cierta secularización, como muchos otros aspectos religiosos, hasta ser concebido como una interacción social que el ser humano necesita para intentar construir su subjetividad y llenar sus vacíos interiores. Evidentemente, no existe communitas si no hay interacción; por tanto, a corto plazo quizás este aspecto resulte perjudicado, pero siendo la dimensión social uno de los elementos mejor apreciados del Camino, no tardará en recuperarse. Por otro lado, este distanciamiento forzoso puede significar más tiempo para estar solo y, por tanto, para pensar y encontrarse, favoreciendo así otro tipo de experiencia característica del Camino: la introspección. De hecho, uno de los atractivos del Camino es el "tiempo", que los peregrinos aprenden a recuperar y a valorar por estar lejos de los ritmos acelerados de la vida cotidiana.

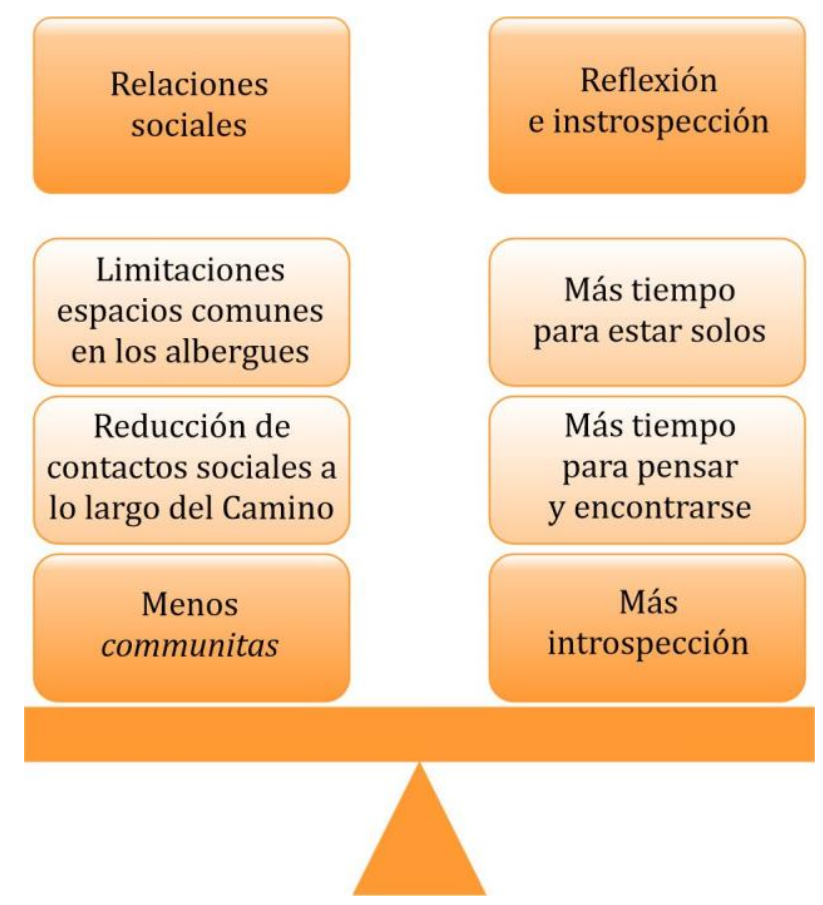

Figura 4. Posibles efectos del distanciamiento social. Fuente: elaboración propia.

\subsection{El Camino, ¿volverá a ser el mismo o sufrirá una "metamorfosis"?}

La movilidad es una peculiaridad de la dimensión interior del peregrino poscontemporáneo (Coleman y Eade, 2004), convirtiendo la peregrinación en modelo de movilidad transnacional (con los dife- 
rentes impactos que esto pueda tener). El 21 de junio de 2020 se declara el cese del estado de alarma en España y el 1 de julio de 2020 se reabre oficialmente el Camino de Santiago. A partir de este momento, empieza una lenta y progresiva reaparición de los peregrinos. Además de las limitaciones higiénico-sanitarias que introdujeron cambios en el mundo de la peregrinación, y de la procedencia de los peregrinos, atendiendo a las fuentes estadísticas de la Oficina del Peregrino, otros cambios se asoman en el Camino. En primer lugar, como ya se ha indicado, desde el punto devista sociodemográfico, en el verano de 2020 disminuye el número de peregrinos con más de 60 años, y en segundo lugar, entre los peregrinos que terminan su Camino en los meses de julio, agosto y septiembre de 2020 disminuye la motivación religiosa. Este cambio puede justificarse gracias a los valores poscontemporáneos asociados al peregino en estos inicios del siglo XXI; es decir, ansia por caminar y por reafirmarse (Lorimer, 2011). Además, contextualizando esos aspectos a un momento histórico pospandemia, el Camino volverá a ser una ocasión para salir, caminar y estar en contacto con la naturaleza y recuperar aquella libertad de movimiento que la pandemia limitó. Esta recuperación podrá ser pautada y dependerá de la distancia y de la procedencia de los caminantes. El Camino ofrece la posibilidad de escapar de la masificación y de disfrutar de los diferentes recursos del entorno natural (Galvani, Lew e Sotelo Pérez, 2020). Quizás será precisamente la motivación holística la que dominará en los próximos años.

Otro aspecto que habría que tener en consideración para entender el futuro del Camino reside en una de las primeras definiciones de peregrinación, originariamente considerada una quest, es decir, un viaje espiritual que comporta la búsqueda de sí mismo (Morinis, 1992; Osterrieth, 1997; Turner y Turner, 1978). Al final de la quest, se produce una nueva identidad o un nuevo estatus; por ello, la experiencia peregrinatoria no termina con la llegada al destino o con la vuelta a casa, porque es una experiencia espiritual que afecta a la percepción del mundo, lleva a profundas experiencias interiores y estimula a repensar el pasado y a tener una visión renovada del futuro (Frey, 1998). El "peregrino de mañana" necesitará esta quest y este momento catártico, por ello podrá encontrar en el Camino una respuesta a sus inquietudes. Por tanto, la ruta jacobea tiene los requisitos de "ruta de terapia possecular", y quizás "posCOVID". Según L. Mikaelsson (2012), la ruta terapéutica está constituida por dos elementos: el primero es la comprensión de heridas personales (como divorcio, muerte, pérdida del trabajo, etc.), el segundo elemento consiste en experiencias de renovación o transformación. Ambos elementos convergen en este momento histórico, que puede hacer del Camino un ejemplo de terapia gradual que traiga beneficios para la curación física, mental y espiritual. Para tal propósito, no hay que olvidar el ya mencionado auge del Camino hacia Fisterra, y que podría renovarse ya que cuenta con la experiencia de otro paisaje: el paisaje marítimo (y el agua como elemento catártico). El paisaje encantador de Fisterra y el propio nombre de la Costa da Morte están acompañados de un gran simbolismo que surge de una localización en una de las regiones más occidentales de Europa, y enlazan con una rica tradición de mitos y leyendas (Alonso, 2002; Blom et al., 2016; Sánchez-Carretero, 2015). Hasta el 2019, los peregrinos continuaban hasta el fin del mundo porque querían regresar a la naturaleza (lejos de la aglomeración urbana de Santiago). En la actualidad, llegar hacia el océano recuperaría su significado catártico y ancestral. La regeneración espiritual es una expectativa de los peregrinos que, cumpliendo antiguos rituales asociados al fuego y al agua, darían un paso más hacia la conceptualización de espiritualidad poscontemporánea (Margry, 2015).

\section{Conclusiones}

La crisis causada por la COVID-19 ofrece la oportunidad de establecer prioridades y direcciones necesarias para apostar por un desarrollo futuro equilibrado, restablecer la visión del futuro a corto y a medio plazo, y revisar activamente estudios e informes anteriores sobre prácticas turísticas insostenibles para evitar su repetición; en definitiva, debería enseñar a hacer las cosas en virtud de nuevos valores, como la equidad, la ética, la inclusión, la sostenibilidad, la responsabilidad, la justicia social y ambiental y, especialmente, la resiliencia (Benjamin et al., 2020; Edelheim, 2020; Fletcher et al., 2020; Gössling et al., 2021; Higgins-Desbiolles, 2020; UNWTO, 2020). Las olas de pandemia muestran la continuidad de los efectos en diferentes sectores de la vida económica, social y cultural, y con ello en los comportamientos de la población (Mróz, 2021; Oxholm et al., 2020). Teniendo en cuenta que el Ca- 
mino ofrece una forma alternativa de viaje sostenible, lejos de los centros concurridos y en pleno contacto con lo rural, este volverá a estar de moda, reconfirmando esquemas de consumo plurales y trasversales.

Las motivaciones del peregrino parecen adecuarse (y actualizarse) según el momento histórico y cultural, interpretando así los paradigmas de nuevas corrientes religiosas, espirituales y seculares (Farias et al., 2018; Moscarelli et al., 2020; Possamai, 2003). Debido a lo vivido en esos meses, las motivaciones podrán ser cada vez más diversas, y quizás aparecerá alguna motivación específicamente relacionada con una "terapia pospandemia", basada en el contacto con la naturaleza y con el aire libre. El Camino volverá a ser una modalidad de redescubrimiento de la naturaleza de la mano del goce de diferentes recursos a lo largo de la ruta, ampliando así su ya variada oferta y aumentando las dificultades en reconocer los límites entre peregrinos o turistas, y con ello la caracterización de un perfil cada vez más complejo. Todo esto requerirá una gestión y planificación de la ruta que establezca prioridades para garantizar un desarrollo sostenible. Será necesaria la máxima coordinación entre los distintos organismos gestores, sin olvidar que la recuperación de la movilidad a lo largo del Camino puede contribuir a la recuperación de las economías locales.

Los planes de gestión mencionados a lo largo del texto confirman el interés en la planificación del Camino. Sus gestores tienen una responsabilidad no solo local, sino también internacional. En cuanto a su modelo de gestión de bioseguridad, podría servir de ejemplo para otros itinerarios. Así como en el pasado, el Camino ha sido un benchmark en la planificación y gestión territorial de un itinerario cultural, podrá volver a serlo en el futuro, pues el Camino acoge numerosos peregrinos y por ello todo el mundo lo mira para aprender.

Desde hace unos meses han empezado diferentes campañas de vacunación, pero el clima de incertidumbre sigue imperando en el escenario internacional. Ha empezado una lucha geopolítica precisamente asociada a las vacunas que, junto a la evolución económica, será la principal responsable de determinar el rumbo de los desplazamientos internacionales. Por tanto, teniendo en cuenta la fecha de finalización de este trabajo ( 7 de junio de 2021), apostamos por continuar monitoreando el desarrollo de la pandemia COVID-19 a lo largo del Camino, atendiendo a los perfiles sociodemográficos y motivacionales de los peregrinos, para valorar qué papel va a desempeñar este tanto en términos socioeconómicos como en términos terapéuticos.

\section{Autoría del trabajo}

Conceptualización, L.L. y R.C.L.G.; metodología, L.L.; adquisición de datos, L.L. y R.C.L. G; análisis e interpretación, L.L.; redacción y preparación del borrador, L.L.; redacción, revisión y edición, L.L. y R.C.L.G. Todos los autores han leído y están de acuerdo con la versión publicada del manuscrito.

\section{Bibliografía}

Alonso, F. (2002). Historias, leyendas y creencias de Finisterre. Betanzos: Briga.

Apollo, M., Wengel, Y., Schänzel, H., y Musa, G. (2020). Hinduism, ecological conservation and public health: What are the health hazards for religious tourists at hindu temples? Religions, 11(8), 416. DOI: https://doi.org/10.3390/rel11080416

Benjamin, S., Dillette, A., y Alderman, D. H. (2020). "We can't return to normal": Committing to tourism equity in the post-pandemic age. Tourism Geographies, 22(3), 476-483. DOI: https://doi.org/10.1080/14616688.2020.1759130

Blom, T., Nilsson, M., y Santos, X. M. (2016). The Way to Santiago beyond Santiago. Fisterra and the pilgrimage's post-secular meaning. European Journal of Tourism Research, 12, 133-146. Recuperado de: https://ejtr.vumk.eu/index.php/about/article/view/217

Cazaux, F. (2011). To be a pilgrim: A contested identity on Saint James' Way. Tourism. Original Scientific Papers, 59(3), 353-367. Recuperado de: https://hrcak.srce.hr/74038

CETUR, y Xacobeo S.A. (2007-2010). Observatorio Estadístico do Camiño de Santiago 2007, 2008,2009 e 2010. Santiago de Compostela: Centro de Estudios Turísticos / Xunta de Galicia.

Chemin, E. (2011). Pilgrimage in a secular age: Religious \& consumer landscapes of late modernity. (Unpublished doctoral thesis). University of Exeter. Recuperado de: http://hdl.handle.net/10036/3672 
Clúster Turismo de Galicia. (2020). Revisións de impacto do Covid-19 no sector turístico gallego. Santiago de Compostela: Clúster Turismo de Galicia. Recuperado de:

https://clusterturismogalicia.com/wpcontent/uploads/2020/04/2020 04_14_Previsión_COVID_v4_FINAL.pdf

Cohen, E. (1992). Pilgrimage and tourism: Convergence and divergence. En A. Morinis (Ed.), Sacred journeys. The anthropology of pilgrimage (pp. 47-61). Westport, CT: Greenwood Press.

Coleman, S., y Eade, J. (2004). Reframing pilgrimage. Cultures in motion. London, UK/New York, NY: Routledge.

Collins-Kreiner, N. (2010). The geography of pilgrimage and tourism: Transformations and implications for applied geography. Applied Geography, 30(1), 153-164. DOI: https://doi.org/10.1016/j.apgeog.2009.02.001

Collins-Kreiner, N. (2015). Dark tourism as/is pilgrimage. Current Issues in Tourism, 19(12), 1185-1189. DOI: https://doi.org/10.1080/13683500.2015.1078299

Creswell, J.W. (1998). Qualitative research inquiry and research design. Choosing among five traditions. Thousand Oaks, CA: Sage.

Creswell, J. W. (2014). Research design: qualitative, quantitative and mixed methods approaches. Los Angeles, CA: Sage.

Denzin, N.K., y Lincoln, Y.S. (2018). The sage handbook of qualitative research. Los Angeles, CA/London, UK: Sage.

Digance, J. (2003). Pilgrimage at contested sites. Annals of Tourism Research, 30(1), 143-159. DOI: https://doi.org/10.1016/S0160-7383(02)00028-2

Doi, K. (2011). Onto emerging ground: Anticlimactic movement on the Camino de Santiago de Compostela. Tourism. Original Scientific Papers, 59(3), 271-285. Recuperado de: https://hrcak.srce.hr/74033

Eade, J., y Sallnow, M. (1991). Contesting the sacred. The anthropology of christian pilgrimage. London, UK/New York, NY: Routledge.

Edelheim, J. (2020). How should tourism education values be transformed after 2020? Tourism Geographies, 22(3), 547-554. DOI: https://doi.org/10.1080/14616688.2020.1760927

Farias, M., Coleman, T. J., Bartlett, J. E., Oviedo, L., Soares, P., Santos, T., y Bas, M. C. (2018). Atheists on the Santiago Way: Examining motivations to go on pilgrimage. Sociology of Religion, 80(1), 28-44. DOI: https://doi.org/10.1093/socrel/sry019

FEAACS. (2020). Toca repensar el Camino de Santiago. Logroño: Federación Española de Asociaciones de Amigos del Camino de Santiago. Recuperado de: https://www.caminosantiago.org/cpperegrino/prensa/verprensa.asp?PrensaID=15157\&fbclid=IwAR0qFQ4 iYteR6yN306PFwi0w5bT4p6HGVki2e2gKFSpYtdruxWuVCvIsl8c.

Febrero Paños, E., y Bermejo Patón, F. (2021). Estimating the impact of COVID-19 on the Spanish economy with input-output analysis. Revista Galega de Economía, 30(1), 1-19. DOI: http://dx.doi.org/10.15304/rge.30.1.7105

Fernández Fernández, M., Fernández Méndez, D., y Riveiro García, D. (2021). El impacto del Camino de Santiago a escala local: la percepción de los residentes de dos municipios gallego. Cuadernos de Turismo, 47, 37-63. DOI: https://doi.org/10.6018/turismo.474001

Fletcher, R., Murray Mas, I., Blázquez-Salom, M., y Blanco-Romero, A. (2020). Tourism, degrowth, and the COVID19 crisis. POLLEN Political Ecology Network. Recuperado de: https://politicalecologynetwork.org/2020/03/24/tourism-degrowth-and-the-covid-19-crisis

Fraternidad Internacional del Camino de Santiago. (2014). Manifiesto de Villafranca del Bierzo. I Foro Internacional "El Legado de Elías Valiña". Villafranca del Bierzo. 14 de diciembre de 2014. Santiago de Compostela: Fraternidad Internacional del Camino de Santiago.

Recuperado de: http://www.xacobeo.fr/ZF1.05.gest Villafranca-2014 es.pdf

Frey, N. L. (1998). Pilgrim stories: On and off the road to Santiago. Berkeley, CA/London, UK: University of California Press.

Friedman, T. L. (17 de marzo de 2020). Our new historical divide: B.C. and A.C.- The world before corona and the world after. Here are some trends to watch. The New York Times.

Recuperado de: https://www.nytimes.com/2020/03/17/opinion/coronavirus-trends.html

Galvani, A., Lew, A. A., y Sotelo Pérez, M. (2020). COVID-19 is expanding global consciousness and the sustainability of travel and tourism. Tourism Geographies, 22(3), 567-576.

DOI: https://doi.org/10.1080/14616688.2020.1760924

Gesler, W. (1996). Lourdes: Healing in a place of pilgrimage. Health and Place, 2(2), 95-105. DOI: https://doi.org/10.1016/1353-8292(96)00004-4

Gössling, S., Scott, D., y Hall, C. M. (2021). Pandemics, tourism and global change: a rapid assessment of COVID-19. Journal of Sustainable Tourism, 29(1), 1-20. DOI: https://doi.org/10.1080/09669582.2020.1758708

Harvey, D. (19 de marzo de 2020). Anti-capitalist politics in the time of COVID-19. David Harvey The AntiCapitalist Chronicles.

Recuperado de: http://davidharvey.org/2020/03/anti-capitalist-politics-in-the-time-of-covid-19/ 
Higgins-Desbiolles, F. (2020). Socialising tourism for social and ecological justice after COVID-19. Tourism Geographies, 22(3), 610-623. DOI: https://doi.org/10.1080/14616688.2020.1757748

Kurrant, C. (2019). Biographical motivations of pilgrims on the Camino de Santiago. International Journal of Religious Tourism and Pilgrimage, 7(2), 3. DOI: https://doi.org/10.21427/06p1-9w68

Lois González, R.C. (2013). The Camino de Santiago and its contemporary renewal: Pilgrims, tourists and territorial identities. Culture and Religion, 14(1), 8-22. DOI: https://doi.org/10.1080/14755610.2012.756406

Lois González, R. C., Castro Fernández, B. M., y Lopez, L. (2016). From sacred place to monumental space: The mobility along the way to St. James. Mobilities, 11(5), 770-788.

DOI: https://doi.org/10.1080/17450101.2015.1080528

Lois González, R. C., y Lopez, L. (2012). El Camino de Santiago: una aproximación a su carácter polisémico desde la geografía cultural y el turismo. Documents d'Anàlisi Geogràfica, 58(3), 459-479.

DOI: https://doi.org/10.5565/rev/dag.6

Lois González, R. C., y Santos, X. M. (2015). Tourists and pilgrims on their way to Santiago. Motives, caminos and final destinations. Journal of Tourism and Cultural Change, 13(2), 149-164.

DOI: https://doi.org/10.1080/14766825.2014.918985

Lopez, L. (2020). Representing and performing pilgrimage in a comic book: On the Camino. International Journal of Religious Tourism and Pilgrimage, 8(5), 4.

Recuperado de: https://arrow.tudublin.ie/cgi/viewcontent.cgi?article=1407\&context=ijrtp

Lopez, L., y Lois González, R. C. (2020). New tourism dynamics along the Way of St. James. From undertourism and overtourism to the post-COVID-19 era. En G. X. Pons, A. Blanco-Romero, R. Navalón-García, L. Troitiño-Torralba y M. Blázquez-Salom (Eds.), Sostenibilidad turística: overtourism vs undertourism. Mon. Soc. Hist. Nat. Balears, 31. (pp. 541-552). Palma de Mallorca: Societat d'Història Natural de les Balears.

Recuperado de: https://www.ucm.es/geoturis/file/sostenibilidad-turistica-tot mendoza?ver

Lorimer, H. (2011). Walking: New forms and spaces for studies of pedestrian. In T. Cresswell y P. Merriman (Eds.), Geographies of mobilities: Practices, spaces, subjects (pp. 19-35). Farham, UK: Ashgate.

Losada Sánchez, M. N., Alén González, M. E., y Domínguez Vila, T. (2014). Aproximación ao perfil e ao comportamento do turista senior. Revista Galega de Economía, 23(2), 69-90.

DOI: https://doi.org/10.15304/rge.23.2.2483

Margry, P. J. (2015). To be or not to be... A pilgrim. Spiritual pluralism along the Camino Finisterre and the urge for the end. En C. Sánchez-Carretero (Ed.), Heritage, pilgrimage and the Camino to Finisterre. Walking to the end of the world (pp. 175-211). Heidelberg,Germany/New York, NY/Dordrecht, Netherlands/London, UK: Springer.

Martínez Roget, F., Castro Domínguez, M. N., y Fraiz Brea, J. A. (2018). 0 turismo como fonte de riqueza en Galicia: análise dalgunhas experiencias. Revista Galega de Economía, 27(3), 65-80.

DOI: https://doi.org/10.15304/rge.27.3.5437

Martín-Duque, C. (2017). Los impactos del turismo en el Camino de Santiago Francés: una aproximación cualitativa. Methaodos. Revista de Ciencias Sociales, 5(1), 62-73. DOI: https://doi.org/10.17502/m.rcs.v5i1.155

Mason, J. (1996). Qualitative Researching. Thousand Oaks, CA: Sage.

Mikaelsson, L. (2012). Pilgrimage as post-secular therapy. Scripta Instituti Donneriani Aboensis, 24. DOI: https://doi.org/10.30674/scripta.67418

Milani, R. (2001). L'arte del paesaggio. Padova, Italia: Il Mulino.

Morinis, A. (1992). Sacred journeys. The anthropology of pilgrimage. Westport, CT: Greenwood Press.

Moscarelli, R., Lopez, L., y Lois González, R. C. (2020). Who is interested in developing the Way of Saint James? The pilgrimage from faith to tourism. Religions, 11(1), 24. DOI: https://doi.org/10.3390/rel11010024

Mróz, F. (2021). The impact of COVID-19 on pilgrimages and religious. Tourism in Europe during the first six months of the pandemic. Journal of Religious Health, 60, 625-645.

DOI: https://doi.org/10.1007/s10943-021-01201-0

Nilsson, M., y Tesfahuney, M. (2019). Pilgrimage mobilities: a de Certeauian perspective. Geografiska Annaler: Series B, Human Geography, 101(3), 219-230. DOI: https://doi.org/10.1080/04353684.2019.1658535

Oficina del Peregrino. (2009-2020). Informes estadísticos 2009-2020. Santiago de Compostela: Oficina del Peregrino. Recuperado de: www.archicompostela.org.

Osterrieth, A. (1997). Pilgrimage, travel and existential quest. En R. H. Stoddard y A. Morinis (Eds.), Sacred places, sacred spaces. The geography of pilgrimage (pp. 25-39). Baton Rouge, LA: Geoscience.

Oxholm, T., Rivera, C., Schirrman, K., y Hoverd, W. J. (2020). New Zealand religious community responses to COVID-19 while under level 4 lockdown. Journal of Religion and Health, 60, 16-33.

DOI: https://doi.org/10.1007/s10943-020-01110-8

Patton, M. (2002). Qualitative research and evaluation methods (3rd ed.). Thousand Oaks, CA: Sage. 
Porcal Gonzalo, M. C., Díez Angulo, A., e Íñiguez de Heredia, J. J. (2012). Dimensión territorial y turística de la ruta norte del Camino de Santiago en el País Vasco: distintas concepciones, valoraciones y propuestas de intervención sobre un fenómeno multifacético. Boletín de la Asociación de Geógrafos Españoles, 58, 177-204.

DOI: https://doi.org/10.21138/bage.2064

Possamai, A. (2003). Alternative spiritualties and the cultural logic of late capitalism. Culture and Religion, 4(1), 31-45. DOI: https://doi.org/10.1080/01438300302807

Riveiro García, D. (2021). El Impacto del Covid-19 en el perfil del peregrino. Santiago de Compostela: Universidade de Santiago de Compostela, Cátedra do Camiño de Santiago e das Peregrinacións. Recuperado de: https://www.catedradelcaminodesantiago.com/media/uploads/1607611861 INFORME RESULTADOS PRO YECTO Dolores Riveiro.pdf

Sánchez-Carretero, C. (2015). To walk and to be walked...At the end of the world. En C. Sánchez-Carretero (Ed.), Heritage, pilgrimage and the Camino to Finisterre. Walking to the end of the world (pp. 1-20). Heidelberg,Germany/New York, NY/Dordrecht, Netherlands/London, UK: Springer

Santos, X. M., y Lois González, R. C. (2011). El Camino de Santiago en el contexto de los nuevos turismos. Estudios Turísticos, 189, 95-116. Recuperado de: https://dialnet.unirioja.es/servlet/articulo?codigo=3898981

Sobrino, M. L., y López, F. (Eds.). (2006). Nuevas visiones del paisaje: la vertiente atlántica. Santiago de Compostela: Xunta de Galicia, Consellería de Cultura e Deportes.

Stoddard, R. H., y Morinis, A. (Eds.). (1997). Sacred places, sacred spaces. The geography of pilgrimage. Baton Rouge, LA: Geoscience.

Timothy, D., y Olsen, D. (Eds.). (2006). Tourism, religion and spiritual journeys. London, UK: Routledge.

Tobler, W. (1970). A computer model simulation of urban growth in the Detroit region. Economic Geography, 46(2), 234-240. Recuperado de: https://www.jstor.org/stable/143141

Turner, V., y Turner, E. (1978). Image and pilgrimage in christian culture. New York, NY: Colombia University Press.

UNWTO. (2020). Supporting jobs and economies through travel and tourism. A call for action to mitigate the socioeconomic impact of COVID-19 and accelerate recovery. Madrid: World Tourism Organization. Recuperado de: https://webunwto.s3.eu-west-1.amazonaws.com/s3fs-public/202004/COVID19 Recommendations English 1.pdf

Vasilachis, I. (2006). Estrategias de investigación cualitativa. Barcelona: Gedisa.

Xunta de Galicia. (2015). Plan director y estratégico del Camino de Santiago en Galicia 2015-2021. Santiago de Compostela: Xunta de Galicia. Recuperado de: https://www.turismo.gal/canle-profesional/plans-e-proxectos/plan-director-camino-desantiago?langld=es ES\#: :text=El\%20Plan\%20Director\%20del\%20Camino,privados\%2C\%20implicados\%20en\% 20el\%20mismo

Xunta de Galicia. (2019). Plan estratégico del Xacobeo 2021. Santiago de Compostela: Xunta de Galicia. Recuperado de: https://www.turismo.gal/osdam/filestore/1/8/0/5/3 66c1f535732692d/18053 e66ed12a82097c3.pdf

Xunta de Galicia. (2020). Plan de reactivación de los sectores cultural y turístico frente a los efectos derivados de la COVID-19. Santiago de Compostela: Xunta de Galicia. Recuperado de: https://xacobeo2021.caminodesantiago.gal/es/institucional/plan-de-reactivacion-de-los-sectores-culturaly-turistico 